\title{
Retroperitoneoscopic renal and adrenal specimen resection surgery in children
}

\author{
Liang Zhong, Xiangyu Zou, Jie Sun \\ Department of Urology, Shanghai Children's Medical Center, Shanghai Jiao Tong University, School of Medicine, Shanghai, China \\ Videosurgery Miniinv 2021; 16 (1): 256-263 \\ DOI: https://doi.org/10.5114/wiitm.2020.94923
}

\begin{abstract}
Introduction: The most common indication for retroperitoneoscopy in children is poorly functioning kidney, related to reflux or obstruction. Few pediatric urologists attempt to conduct a mass resection operation under retroperitoneoscopy, especially in infants with oncological diseases.

Aim: In this study, we describe our experience in a series of resection operations under retroperitoneoscopy to demonstrate its safety and efficacy in children, especially in the treatment of pediatric urology tumor.

Material and methods: A retrospective review of 18 consecutive retroperitoneoscopic resection operations from December 2017 to July 2019 was made. Then a comparison study between retroperitoneoscopy and transperitoneal laparoscopy for exclusively oncological patients was conducted.

Results: Retroperitoneoscopic surgery was successfully performed on 18 patients, 11 having solid tumors, 2 with cysts, and 5 with nonfunctioning or poorly functioning kidneys. The mean age was $69.5 \pm 46.9$ months. The mean operative time was $138.6 \pm 57.7 \mathrm{~min}$, while the mean size of the mass was $6.4 \pm 3.8 \mathrm{~cm}$ in the largest diameter. Two patients were converted to open surgery because of large diameter of the mass. The 11 solid tumors which were operated on by retroperitoneoscopic surgery were then compared with 13 consecutive oncological patients undergoing transperitoneal laparoscopy. Retroperitoneoscopy has a lower transfusion rate $(p<0.05)$ and faster recovery $(p<0.01)$ compared to transperitoneal laparoscopy.

Conclusions: This study demonstrates that the resection operation of a renal or adrenal specimen under retroperitoneoscopy is feasible and safe in children. It is useful not only to treat patients with benign diseases but also in oncological patients. Retroperitoneoscopy is a recommended surgical approach for children.
\end{abstract}

Key words: laparoscopy, children, renal, retroperitoneal space, adrenal.

\section{Introduction}

The application of retroperitoneoscopy in the pediatric urology patient was delayed compared to the adult population. The smaller working space compared to transperitoneal laparoscopy, combined with reversed orientation of the kidney hilus, makes retroperitoneoscopy hard to master. Thus, retroperitoneoscopy is mostly performed in children with benign disease [1-5]. Few studies have concerned the advantages of retroperitoneoscopy in children, especially in the treatment of pediatric oncological patients. In this study, we describe a series of resection operations under retroperitoneoscopy in children to demonstrate its safety and efficacy in the management of nonfunctioning or poorly functioning kidney-kidney cyst, renal carcinoma, adrenocortical carcinoma-adrenal ganglioneuroblastoma-adrenal teratoma, neuroblastoma, Wilms' tumor, and adrenal hemangioma.

\section{Address for correspondence}

Jie Sun, Department of Urology, Shanghai Children's Medical Center, Shanghai Jiao Tong University, School of Medicine, Shanghai, China,

e-mail: sunjie@scmc.com.cn 


\section{Aim}

We describe our experience in a series of resection operations under retroperitoneoscopy to demonstrate that the retroperitoneoscopic resection operation of a renal or adrenal specimen is feasible and safe in children, not only for benign disease but also in oncological patients.

\section{Material and methods}

\section{Patients and data collection}

We retrospectively reviewed 18 consecutive patients on whom retroperitoneoscopy was carried out by one pediatric urologist from December 2017 to July 2019. The data on clinical parameters such as gender, age, operative time, mass diameter and hospitalization duration were collected. To compare the differences between retroperitoneoscopy and transperitoneal laparoscopy in the treatment of oncological patients, clinical parameters of 13 consecutive patients who underwent transperitoneal laparoscopy were collected.

\section{Operative technique}

The retroperitoneoscopy was carried out using the technique described by Xu Zhang [6]. The patient was placed in the totally lateral decubitus position on the operating table. A 2-cm skin incision was first made below the $12^{\text {th }}$ rib in the posterior axillary line. Then a homemade balloon was inflated outside Gerota's fascia to create the retroperitoneal working space. The first trocar was inserted into this $2-\mathrm{cm}$ incision after the balloon expansion. The other two trocars were inserted into the anterior axillary line under the subcostal margin and the midaxillary line above the iliac crest. The dissection began by incising Gerota's fascia longitudinally to enter the perinephric space. The kidney or adrenal masses were carefully resected after being totally dissociated (Photo 1 ).

\section{Results}

Retroperitoneoscopy was performed in $18 \mathrm{pa}$ tients, 11 having solid tumor, 2 with cyst and 5 with a nonfunctioning or poorly functioning kidney. As show in Table I, the mean age was 69.5 \pm 46.9 months (range: $16-156$ ). The mean size of the mass was $6.4 \pm 3.8 \mathrm{~cm}$ (range: 16 to 2 ) in the largest diameter. The mean operative time was $138.6 \pm 57.7 \mathrm{~min}$ (range: 65-245). Mean hospitalization duration from surgery to discharge was 6.3
Lateral decubitus position
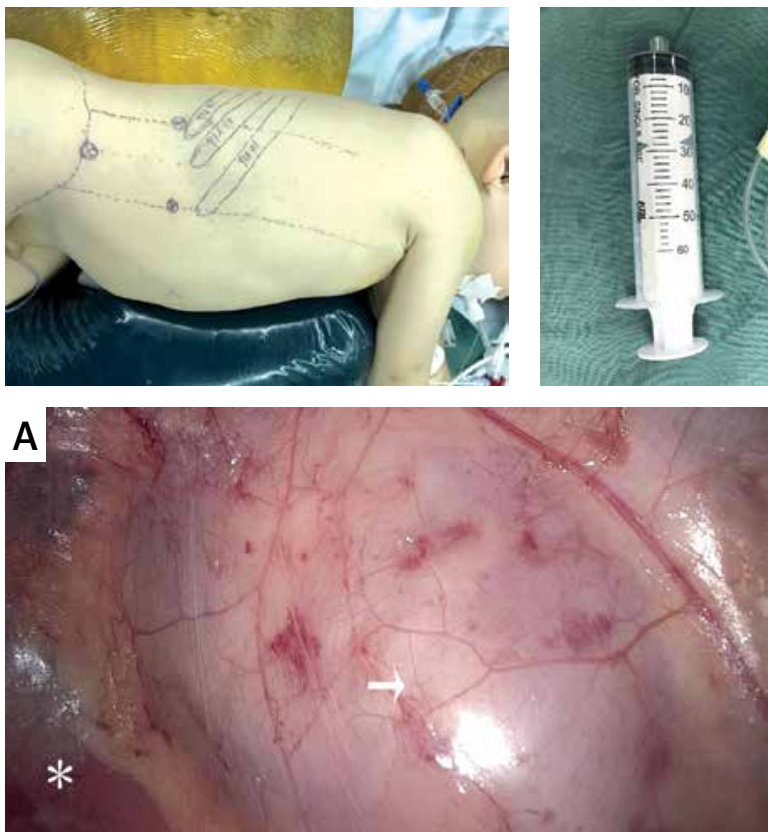

Homemade ballon created using the finger of a glove

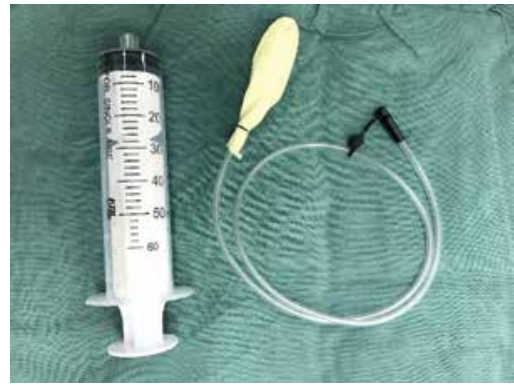

B

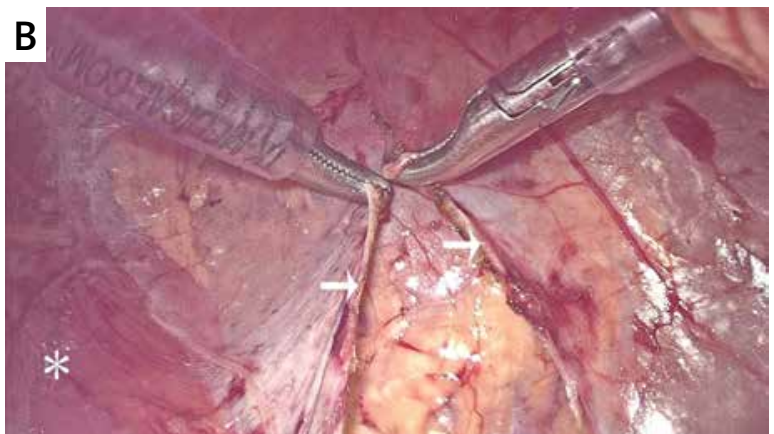

Photo 1. Operation details of the retroperitoneoscopy. Few retroperitoneal (A) and perirenal (B) fat tissue in children facilitated the anatomy under retroperitoneoscopy; psoas major (white asterisk), Gerota's fascia (white arrow) 


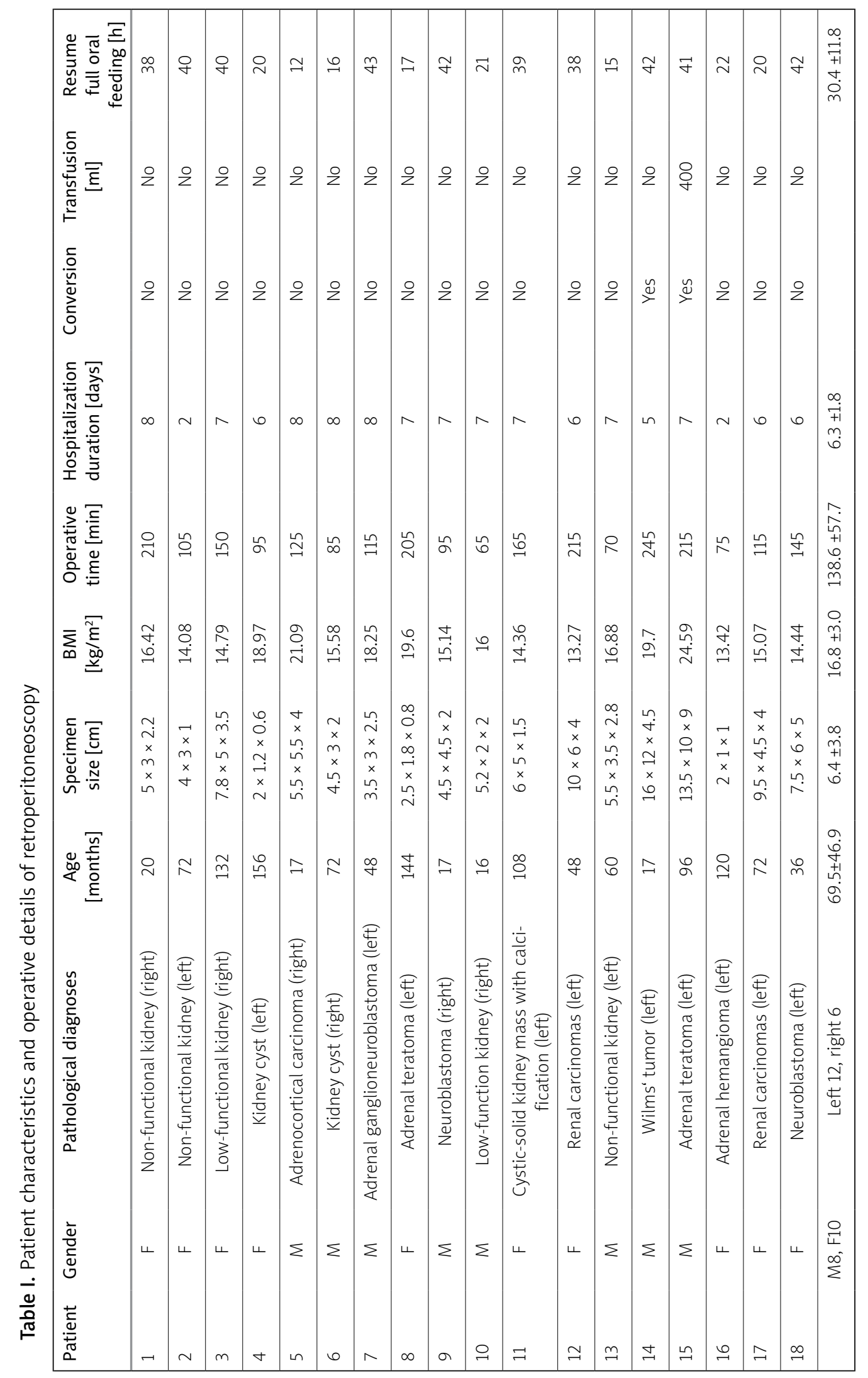




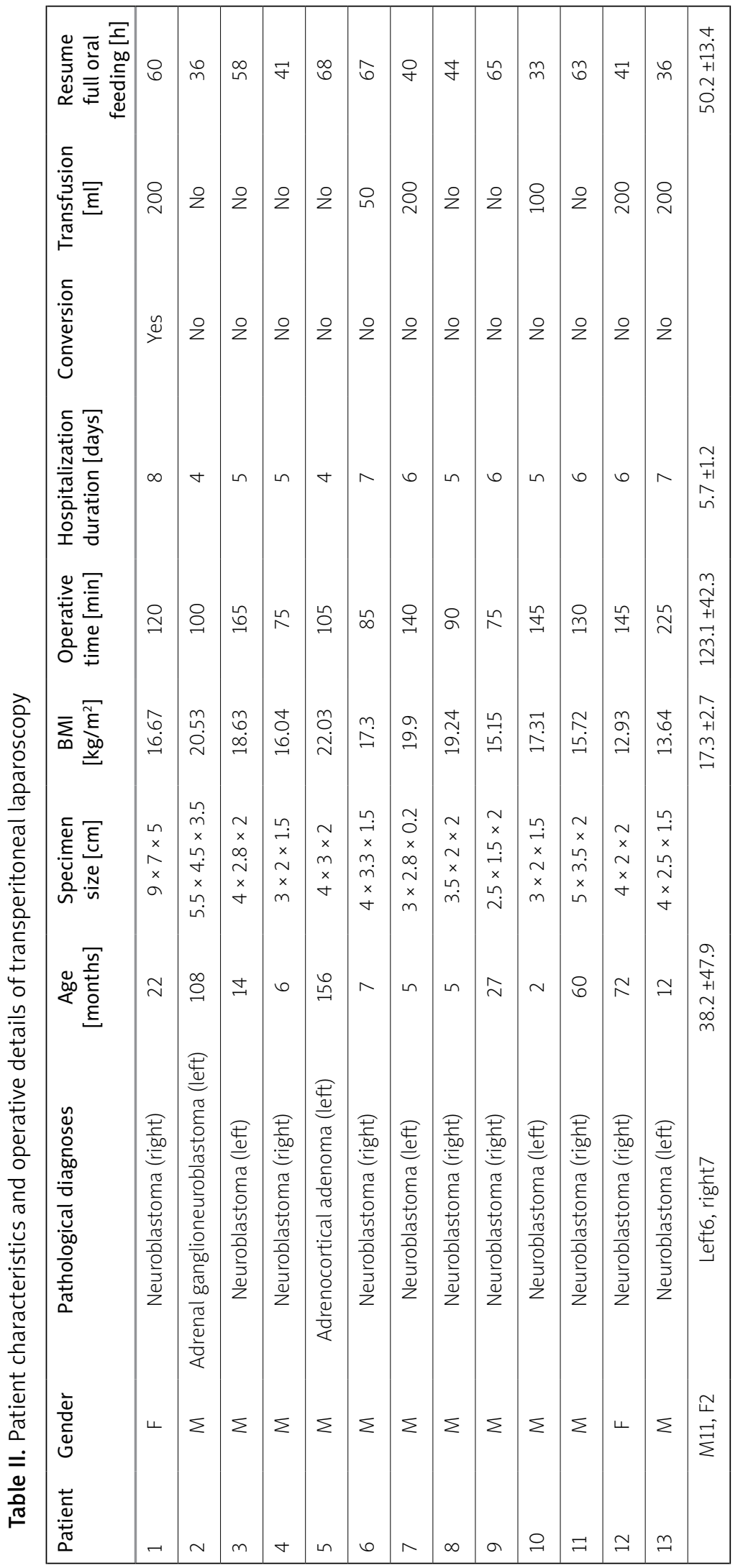


\pm 1.8 days (range: $2-8$ ). The mean time to resume full oral feeding was $30.4 \pm 11.8 \mathrm{~h}$ (range: 12-43). Two patients (patients 14 and 15) were converted to open surgery due to the large diameter of the great mass diameter (16 and $13.5 \mathrm{~cm}$, respectively). Patient 15 received a $400 \mathrm{ml}$ blood transfusion. Pathological diagnoses included two kidney cysts, two renal carcinomas associated with Xp11.2 translocations/TFE3 gene fusion, one adrenocortical carcinoma, one adrenal ganglioneuroblastoma, two adrenal teratomas, two neuroblastomas, one Wilms' tumor, one cystic solid kidney mass with calcification, one adrenal hemangioma and 5 cases of poorly functioning kidney.

Clinical parameters of 13 consecutive oncological patients who underwent transperitoneal laparoscopy were then collected (Table II), which were used to compared with the 11 solid tumor patients in Table I. As shown in Table III, the transfusion rate $(p<0.05)$ and mean time to resume full oral feeding $(p<0.01)$ were significantly lower in retro- peritoneoscopy compared to transperitoneal laparoscopy. No significant differences between the two group were found in regard to age, operative time, hospitalization duration and conversion rate.

\section{Discussion}

Most pediatric urologists prefer transperitoneal laparoscopy because of a wider working space and familiarization with transperitoneal anatomy compared to retroperitoneoscopy. Comparative studies based on adult patients had showed no significant differences in operative time, estimated blood loss, specimen weight, or complication rates [7-9]. However, few pediatric surgeons attempt to conduct a resection operation of kidney or adrenal masses under retroperitoneoscopy, especially in infants with oncological diseases. Hence most of the pediatric studies were based on the application of retroperitoneoscopy in the management of benign disease such as duplex or nonfunctional kidney. According to our

Table III. Statistical analysis of retroperitoneoscopy and transperitoneal laparoscopy in treatment of oncology patients

\begin{tabular}{|c|c|c|c|}
\hline Parameter & $\begin{array}{l}\text { Retroperitoneoscopy } \\
\qquad(N=11)\end{array}$ & $\begin{array}{c}\text { Transperitoneal } \\
\text { laparoscopy }(N=13)\end{array}$ & Statistical analysis \\
\hline Age [months] & $65 \pm 45$ & $38 \pm 47$ & $P>0.05$ ( $t$ test $)$ \\
\hline Male/female & $5 / 6$ & $11 / 2$ & \\
\hline Right/left & $2 / 9$ & $7 / 6$ & \\
\hline \multicolumn{4}{|l|}{ Diagnosis: } \\
\hline Adrenocortical adenoma: & 0 & 1 & \\
\hline Adrenocortical carcinoma & 1 & 0 & \\
\hline Adrenal ganglioneuroblastoma & 1 & 1 & \\
\hline Adrenal teratoma & 2 & 0 & \\
\hline Neuroblastoma & 2 & 11 & \\
\hline Adrenal hemangioma & 1 & 0 & \\
\hline Renal tumor & 4 & 0 & \\
\hline Operative time [min] & $155 \pm 56$ & $123 \pm 42$ & $P>0.05(t$ test $)$ \\
\hline Hospitalization duration [days] & $6 \pm 1$ & $5 \pm 1$ & $P>0.05(t$ test $)$ \\
\hline Mean time to resume full oral feeding [h] & $32 \pm 12$ & $50 \pm 13$ & $P<0.01$ ( $t$ test $)$ \\
\hline Specimen size in largest diameter (CM) & $7 \pm 4$ & $4 \pm 1$ & $P<0.05$ ( $t$ test $)$ \\
\hline Transfusion rate & $1 / 11$ & $6 / 13$ & $P<0.05\left(\chi^{2}\right.$ test $)$ \\
\hline Conversion to open surgery rate & $2 / 11$ & $1 / 13$ & $P>0.05$ ( $\chi^{2}$ test $)$ \\
\hline
\end{tabular}



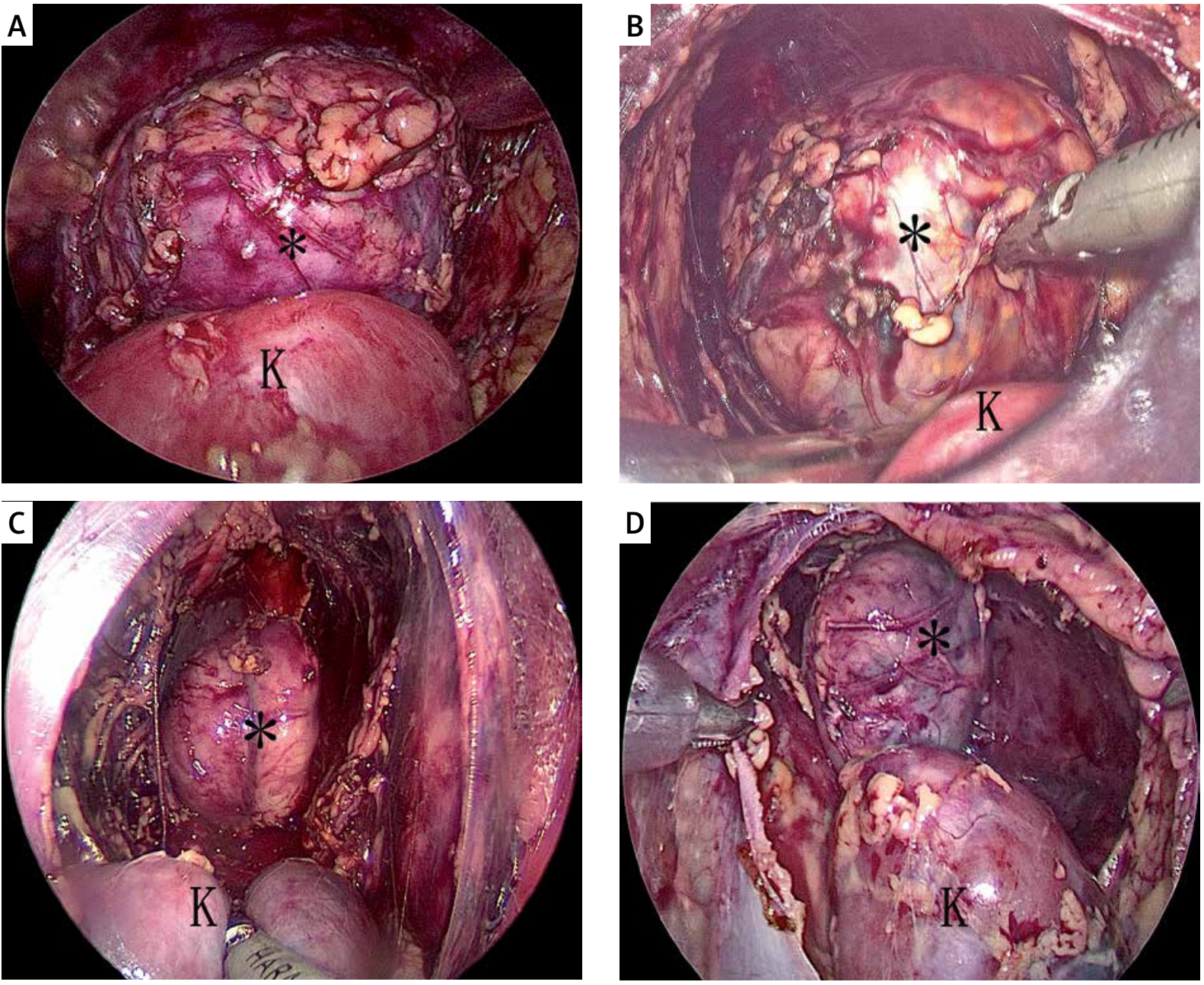

Photo 2. The lateral decubitus position provides direct access to the adrenal and kidney. A, B, C, D are patients 18, 5, 7, 9 respectively. Adrenal mass (black asterisk), kidney (black K)

findings in this study, retroperitoneoscopic resection operation of a renal or adrenal specimen is feasible and safe in children, not only for benign disease but also in oncological patients.

Some surgeons prefer to conduct retroperitoneoscopic procedures on patients lying in the prone position [10-12]. The patient lies in the prone position with pads under the chest and pelvis so as to create a working retroperitoneoscopic space. Although there is no significant difference in operative time between the lateral and posterior approaches, the prone position may affect the breathing of the children and reduce their lung capacity. In our opinion, the totally lateral decubitus position can avoid this problem and provide direct access to the adrenal gland and the kidney. This greatly reduces the potential injury to intra-abdominal organs. As shown in Photos $2 \mathrm{~A}-\mathrm{D}$, the adrenal masses are safe to dissociate and resect. There was almost no damage to the intra-abdominal organs if all the procedures were conducted within Gerota's fascia. However, the risk of conversion to open surgery increased with the growth of the specimen's diameter, because the narrow working space limits the procedure. Hence, retroperitoneoscopy is not recommended for large specimens.

Another advantage of retroperitoneoscopy in children is that it provides easy visualization of the adrenal vein and the kidney vessel compared to the transperitoneal approach. That greatly reduces the risk of bleeding, which is advantageous when dealing with the oncological patient. Dealing with the central adrenal vein is the last step in adrenal surgery. As shown in Photos 3 A and B, it is safe to clip the central adrenal vein on the surface of the vena 

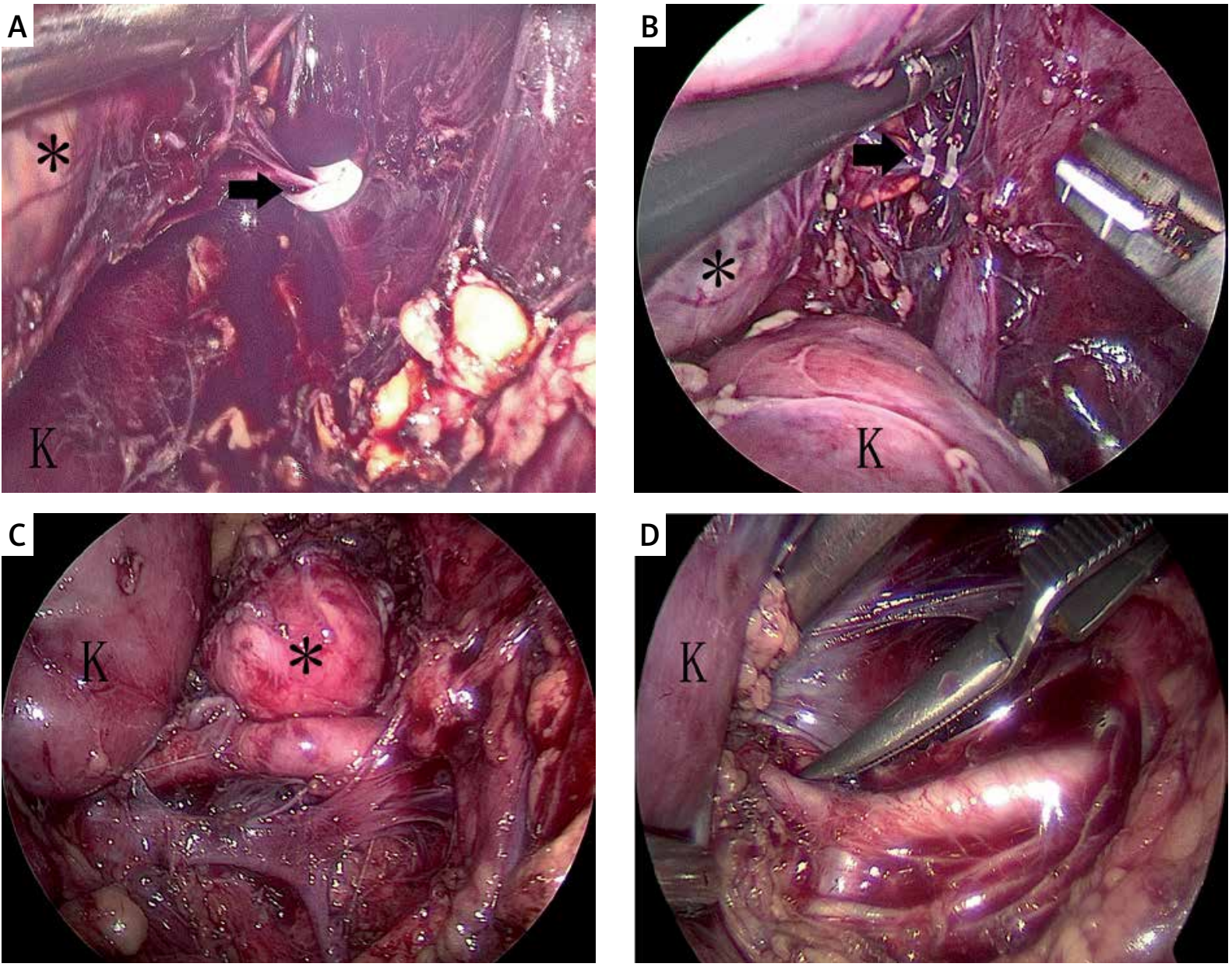

Photo 3. Visualization of adrenal vein and kidney vessel. The central adrenal vein was clipped with a Hem-o-lock (black arrow) in patient 5 (A) and patient 9 (B). C - Renal artery and vein were visualized in patient 8. D - The renal artery branch was dissected and blocked in patient 11. Adrenal mass (black asterisk), kidney (black K)

cava, although both the patients were 17 months old and the largest diameter of the adrenal mass was 5.5 and $4.5 \mathrm{~cm}$, respectively. Photos $3 \mathrm{C}$ and D show the left side of children on whom retroperitoneoscopic surgery was performed. Both the trunk and branches of the renal artery were safe to visualize when dissected on the surface of the psoas major. The left genital vein and lumbar vein, which drain into the left renal vein, can also be dissected safely in children (Photos $3 \mathrm{C}$ ). The transfusion rate, which is significantly lower in retroperitoneoscopy, further confirmed that it is safer in the treatment of the oncological patient than transperitoneal laparoscopy. Moreover, the mean time to resume full oral feeding, which is also significantly shorter in retroperitoneoscopy, demonstrated that the recovery is faster than in the group of transperitoneal laparoscopy.

\section{Conclusions}

This study demonstrates that the resection operation of kidney or adrenal masses under retroperitoneoscopy is feasible and safe for children, especially in infants with oncological diseases. A control study on a large series is needed to corroborate our findings.

\section{Acknowledgments}

This study is supperted by the National Natural Science Foundation of China 81601267 and 81871149.

\section{Conflict of interest}

The authors declare no conflict of interest. 


\section{References}

1. Kim C, McKay K, Docimo SG. Laparoscopic nephrectomy in children: systematic review of transperitoneal and retroperitoneal approaches. Urology 2009; 73: 280-4.

2. Esposito C, Valla JS, Yeung CK. Current indications for laparoscopy and retroperitoneoscopy in pediatric urology. Surg Endosc 2004; 18: 1559-64.

3. Cerulo M, Escolino M, Turra F, et al. Benefits of retroperitoneoscopic surgery in pediatric urology. Curr Urol Rep 2018; 19: 33.

4. Esposito C, Escolino M, Miyano G, et al. A comparison between laparoscopic and retroperitoneoscopic approach for partial nephrectomy in children with duplex kidney: a multicentric survey. World J Urol 2016; 34: 939-48.

5. Escolino M, Farina A, Turra F, et al. Evaluation and outcome of the distal ureteral stump after nephro-ureterectomy in children. A comparison between laparoscopy and retroperitoneoscopy. J Pediatr Urol 2016; 12: 119.e1-8.

6. Zhang X, Fu B, Lang B, et al. Technique of anatomical retroperitoneoscopic adrenalectomy with report of 800 cases. J Urol 2007; 177: 1254-7.

7. Rubinstein M, Gill IS, Aron M, et al. Prospective, randomized comparison of transperitoneal versus retroperitoneal laparoscopic adrenalectomy. J Urol 2005; 174: 442-5.

8. Mohammadi-Fallah MR, Mehdizadeh A, Badalzadeh A, et al. Comparison of transperitoneal versus retroperitoneal laparoscopic adrenalectomy in a prospective randomized study. J Laparoendosc Adv Surg Tech A 2013; 23: 362-6.

9. Liu W, Wang Y, Zhong Z, et al. Transperitoneal versus retroperitoneal laparoscopic nephroureterectomy in the management of upper urinary tract urothelial carcinoma: a matched-pair comparison based on perioperative outcomes. Surg Endosc 2016; 30: 5537-41.

10. Yankovic F, Undre S, Mushtaq I. Surgical technique: retroperitoneoscopic approach for adrenal masses in children. J Pediatr Urol 2014; 10: 400.e1-2.

11. Cho A, Mushtaq I, Retroperitoneoscopic lower pole heminephrectomy. J Pediatr Urol 2019; 15: 89-90.

12. Urbanowicz W, Wieczorek M, Sulislawski J. Retroperitoneoscopic nephrectomy in the prone position in children (point of technique). Eur Urol 2002; 42: 516-9.

Received: 6.02.2020, accepted: 22.03.2020. 